\title{
Wet-processed n-type OTFTs utilizing highly-stable colloids of a perylene diimide derivative
}

Hyeon-Gu Jeon ${ }^{1 \dagger}$, Naomi Oguma ${ }^{2}$, Naoki Hirata $^{2}$, Musubu Ichikawa $^{1,3 *}$

${ }^{1}$ Interdisciplinary Graduate School of Science and Technology, Shinshu University, 315-1 Tokida, Ueda 386-8567, Japan

${ }^{2}$ Dainichiseika Color \& Chemicals Mfg. Co., Ltd., 1-9-4, Horinouchi, Adachiku, Tokyo 123-8588, Japan

${ }^{3}$ JST-PRESTO, Japan Science and Technology Agency (JST), 4-8-1 Honcho, Kawaguchi, Saitama 332-0012, Japan

*Corresponding author. Tel.: +8126821 5498; fax: +81268215417

$\dagger$ Present address: Graduate School of Science and Engineering, Ehime University, 3, Bunkyo-cho, Matsuyama, Ehime 790-8577, Japan

E-mail address: musubu@shinshu-u.ac.jp (M. Ichikawa)

\begin{abstract}
Here we propose and demonstrate a new approach to wet-processed organic thinfilm transistors utilizing highly-stable colloids of small molecular organic semiconductors prepared by laser ablation technique. Highly stable N,N'-ditridecyl3,4,9,10-perylenetetracarboxylic diimide (PTCDI-C13) colloids of 0.1 wt $\%$ concentration were prepared successfully by laser ablation technique in acetonitrile. Mean particle size of the prepared PTCDI-C13 colloid measured by dynamic light scattering method was smaller than $50 \mathrm{~nm}$ which is also confirmed by a transmission electron microscope observation. By simple drop-casting of prepared PTCDI-C13
\end{abstract}


colloids, typical n-type OTFTs with good saturation properties were fabricated successfully. The highest electron mobility was $1.1 \times 10^{-3} \mathrm{~cm}^{2} / \mathrm{Vs}$ and increased up to $0.027 \mathrm{~cm}^{2} / \mathrm{Vs}$ by post-annealing treatment.

Keywords: colloid, wet-process, nanoparticle, laser ablation, PTCDI, OTFT

\section{Introduction}

During the last two decades, organic thin-film transistor (OTFT) has attracted considerable interests due to its strong potential for applications to next-generation electronic devices such as flexible displays and radio frequency identification (RF-ID) tags, and significant progress has been achieved with plenty of publications [1-6]. There are already many reports of OTFTs having higher carrier mobility than amorphous silicon transistors $[7,8]$. Almost all of top records of OTFT performance, however, have been achieved with vacuum-deposited films which is not suitable in the viewpoint of the cost issue. Low manufacturing cost is one of the most important potential feature of OTFTs [9-13]. To achieve truly the low manufacturing cost, it is generally accepted that the fabrication of OTFTs by wet-processes is indispensible [9-13]. The problem, however, is that most of small molecular organic semiconductors with high performance are not soluble in common solvent due to their highly conjugated molecular structures and cannot be handled by wet-processes. For that reason, people have tried to use polymers as the active materials utilizing their high solubility $[14,15]$, or to use precursor molecules for the formation of the active film [16-19], or to synthesis new soluble materials [20-24]. There seems, however, to be performance limits or serious 
demerits in those approaches although the performance of wet-processed OTFTs has been improved rapidly so far. For example, the crystallization of polymer is usually restricted by the long chain structure, and consequently the low crystallinity and the small crystalline grains of polymer films highly limit the carrier mobility [14,15,25-27]. Substitution of solubilizing groups on highly conjugated backbone is usually accompanied by unintended and unfavorable change in molecular-packing pattern by distorting the molecular backbone or by steric hindrance of huge substituents, which results in limited device performance [20-24,28-31]. Therefore, it is highly desirable to make OTFTs with small molecular organic semiconductors without any molecular modification nor performance decrease using some simple wet-processes.

In this paper, we propose and demonstrate a new approach to realize wet-processes of small molecular OTFTs utilizing highly-stable colloids of a perylene diimide derivative prepared by laser ablation technique. It is obvious that we can handle a small molecular organic semiconductor with proper wet-processes if we can make its colloids even though it is not soluble in any solvents.

To realize this new approach, we must clear two important issues. One is to make stable colloids with sufficiently high concentration for using in wet-processes. The stability of colloids, especially, is very important for the uniformity and the reproducibility of device performance. In this work, we have succeeded in making highly stable N,N'-ditridecyl-3,4,9,10-perylenetetracarboxylic diimide (PTCDI-C13, Fig. 1 a) colloids with very high concentration of $0.1 \mathrm{wt} \%$ in acetonitrile using laser ablation technique.

The other is that the grain size of a wet-processed film with colloids will be identical to the particle size of colloids; from several nanometers to dozens of 
nanometers. In that case, there will be huge amount of grain boundaries and we cannot expect high device performance because grain boundaries always play negative roles in the performance of OTFTs. Therefore, it is indispensable to increase the grain size of wet-processed films by proper post-treatment. In this paper, we report that the grain size of PTCDI-C13 films prepared by drop-casting of its colloids was increased effectively by post-annealing treatment which is resulted in the increased electron mobility from $1.1 \times 10^{-3} \mathrm{~cm}^{2} / \mathrm{Vs}$ to $0.027 \mathrm{~cm}^{2} / \mathrm{Vs}$ after post-annealing treatment.

\section{Experimental}

\subsection{Preparation of colloids}

PTCDI-C13 was purchased from Aldrich (95\%) and used without further purification. Acetonitrile was purchased from Aldrich (dehydrated, 99.8\%). PTCDI-C13 of $0.01,0.05$, and $0.1 \mathrm{wt} \%$ was added in acetonitrile and mixed by sonication for $1 \mathrm{~h}$. Laser ablation sample was prepared by putting $3 \mathrm{ml}$ PTCDI-C13/acetonitrile mixture together with a magnetic stirring bar into a quartz cell with the optical path length of 10 mm. Nd:YAG nano-second pulse laser (TII LS-2134, LOTIS) was used for irradiation of laser pulses of the wavelength of $532 \mathrm{~nm}$. The pulse repetition rate was $15 \mathrm{~Hz}$ with the pulse energy of $100 \mathrm{~mJ} / \mathrm{cm}^{2}$. Irradiation time was $10 \mathrm{~m}, 30 \mathrm{~m}$ and $60 \mathrm{~m}$ for 0.01 , 0.05 and $0.1 \mathrm{wt} \%$ mixtures, respectively. UV-vis absorption spectra of prepared colloids were measured with a UV-vis spectrometer (UV-3150, Shimadzu). The size distribution of colloidal particles and the zeta-potential of colloids were measured using Zetasizer Nano ZS (Malvern Instruments). The shape of colloidal particles was observed by 
transmission electron microscope (TEM, H-7650, Hitachi) measurement.

\subsection{OTFT device fabrication}

OTFTs were fabricated in a bottom-contact geometry on heavily doped p-type silicon substrates with a thermally grown $\mathrm{SiO}_{2}$ surface (200 $\mathrm{nm}$ in thickness) that acted as a gate electrode and a gate dielectric (specific capacitance of $18 \mathrm{nF} / \mathrm{cm}^{2}$ ), respectively. The source and drain electrodes (150-nm-thick ITO) were patterned by wet etching (commercial ITO etchant of ITO-02, Kanto Chemicals) an ITO film that was deposited on the $\mathrm{SiO}_{2}$ surface by sputtering after photolithography with a commercial photoresist (THMR-iP 3300, Tokyo Ohka Kogyo). The channel lengths were $10 \mu \mathrm{m}, 20 \mu \mathrm{m}$, and 30 $\mu \mathrm{m}$, which are indicated as the same numbers in the legend in Fig. 5 and Fig. S3. The channel widths were $100 \mu \mathrm{m}, 200 \mu \mathrm{m}$, and $300 \mu \mathrm{m}$, respectively. The substrates were then cleaned by sonication in acetone and isopropyl alcohol (IPA) for $30 \mathrm{~min}$. Then, the substrates were treated by octadecyltrichlorosilane (ODTS, Wako) by immersing in 0.1 M ODTS/toluene solution for $10 \mathrm{~m}$ at $40^{\circ} \mathrm{C}$. Two or five drops of $1 \mu \mathrm{lof} 0.1 \mathrm{wt} \%$ PTCDI-C13 colloid were dropped on electrode-patterned substrates in a nitrogen-filled globe box and dried in $50^{\circ} \mathrm{C}$ vacuum oven for over $1 \mathrm{~h}$. Post-annealing treatment of deposited films was performed at a designated temperature $\left(100^{\circ} \mathrm{C}-180^{\circ} \mathrm{C}\right)$ on a hot plate in a nitrogen environment for $1 \mathrm{~h}$.

\subsection{Characterization}

The surface morphology of the deposited films was examined with an atomic force 
microscope (AFM, Dimension ICON, Veeco) operating in a tapping mode. X-ray diffraction (XRD, Rotaflex, Rigaku) measurements were performed using $\mathrm{Cu} K \alpha(\lambda=$ $1.5418 \AA$ ) radiation under $\theta / 2 \theta$ geometry.

TFT characteristics were measured in a dark high-vacuum chamber at room temperature using a semiconductor device analyzer (B1500A, Agilent Technologies). The field-effect electron mobility $\boldsymbol{\mu}$ was calculated from the saturation regime using the equation

$$
I_{D}=W C_{t} h\left(V_{G}-V_{T}\right)^{2} / 2 L
$$

where $I_{D}$ is the saturated drain current, $W$ and $L$ are the channel width and length, respectively, $C_{i}$ is the capacitance of the gate insulator, $V_{G}$ is the voltage applied to the gate electrode, and $V_{T}$ is the threshold voltage.

\section{Result and discussion}

\subsection{Preparation of PTCDI-C13 colloids}

PTCDI-C13 is a well known n-type material with fairly high electron mobility [3235]. It, especially, has several phase transitions at relatively low temperature due to the long side alkyl chains, and the morphology of vacuum deposited films is largely changed by post-annealing treatment which also leads to the large change in the mobility of its devices [32,36,37]. It is, however, not soluble in common solvents and cannot be handled by wet-processes. In this work, we have tried to prepare highly stable PTCDI-C13 colloids in acetonitrile by laser ablation technique. Preparation of organic nanoparticle colloids by laser ablation technique is well established for various organic 
pigments [38-40]. In addition, one of the authors has already reported that nanoparticle films deposited from organic semiconductor colloids prepared by laser ablation technique can be used for evaluation of electronic properties of organic nanoparticles and also for application to electronic devices such as organic photovoltaic cells [41-43]. We have succeeded to prepare highly stable PTCDI-C13 colloids in this work by using acetonitrile not water as the dispersant as shown in Fig. 1 (b). We could not observe obvious decomposition of PTCDI-C13 in acetonitrile during the laser irradiation although hydrolysis was clearly observed in water (S1). The change of absorption spectra of a $0.01 \mathrm{wt} \%$ PTCDI-C13 acetonitrile colloid is also shown in Fig. 1 (c). Before laser irradiation, most of PTCDI-C13 crystalline particles settle at the bottom of the quartz cell (before irradiation in Fig. 1 b) and no distinct absorbance of PTCDI-C13 was measured (bfIrr in Fig. 1 c). After laser irradiation for 10 m, PTCDI-C13 was fragmented into nanoparticles and a semi-transparent colloid of typical red color was obtained (after irradiation in Fig. 1 b). The absorption spectrum of a just prepared colloid (afIrr_soon in Fig. 1 b) was almost not changed even after 10 days passed (afIrr_10d in Fig. 1 b). Decrease of the absorbance at the peak of $470 \mathrm{~nm}$ was only $1.08 \%$ after 10 days (from 1.755 to 1.736). Actually, we could not find any sedimentation even after 1 year which means that prepared PTCDI-C13 acetonitrile colloid is highly stable.

We have also succeeded in preparing stable colloids with higher concentration. By just increase the irradiation time to $30 \mathrm{~m}$ and $60 \mathrm{~m}$, we can make $0.05 \mathrm{wt} \%$ and $0.1 \mathrm{wt} \%$ colloids, respectively, as shown in Fig. 2 (a). When we plotted the absorbance at the peak of $470 \mathrm{~nm}$ as a function of the concentration, we got a straight line which passes through the origin of the coordinates, as shown in Fig. 2 (b), which means that all of 
PTCDI-C13 was changed into nanoparticles in all of the cases. Moreover, those colloids were also so highly stable as no sedimentation was not observed even after 1 year like $0.01 \mathrm{wt} \%$ colloid.

We have checked the size distribution of colloidal particles. The mean particle size was measured as $c a .45 \mathrm{~nm}$ with the standard deviation of $14 \mathrm{~nm}$ (Fig. 3 a, S2). We have also found that the colloidal particles show rod-like shape with a diameter of much smaller than $100 \mathrm{~nm}$ by a TEM measurement, as shown in Fig. 3 (b), which is well matched with the result of DLS measurement. The rod-like shape (and/or wire shape) of particles is a typical crystal shape of PTCDI derivatives where PTDCI molecules are arranged almost perpendicularly to the long direction of crystals $[29,34,37,44-46]$. The zeta-potential was also measured as large as $-26.3 \mathrm{mV}$ with the standard deviation of $10.5 \mathrm{mV}$ (S3). Those results, small particle size and large zeta-potential, can explain the high stability of the prepared colloid.

\subsection{OTFTs fabrication utilizing colloids}

Using prepared $0.1 \mathrm{wt} \%$ PTCDI-C13/acetonitrile colloid, we could make OTFTs by simple drop casting method. One of the results is shown in Fig. 4 (a). Fig. 4 (b) and (c) show representative output characteristics and transfer characteristics of PTCDI-C13 TFTs before post-annealing treatment. In spite of huge amount of grain boundaries in the film as mentioned in Introduction, deposited nanoparticle films show typical nchannel characteristics with good saturation properties (Fig. 4 b). The highest electron mobility of $1.1 \times 10^{-3} \mathrm{~cm}^{2} /$ Vs was obtained although hysteresis is quite large (Fig. $4 \mathrm{c}$ ). This value is very close to that of vacuum deposited PTCDI-C13 films, $5.4 \times 10^{-3} \mathrm{~cm}^{2} / \mathrm{Vs}$ 
[32]. Moreover, the threshold voltages are also very close, $47 \mathrm{~V}$ vs. $44 \mathrm{~V}$. This result means that PTCDI-C13 nanoparticle films deposited by drop-casting method using colloids can be used as good active layers of OTFTs. The large hysteresis, however, suggests that there may be lots of charge traps in both of the active layer and the interface between the active layer and the gate dielectric.

As mentioned in previous section, the morphology of PTCDI-C13 films can be largely changed by post-annealing treatment which leads to large enhancement of the device performance. We have checked if the morphology and the device performance of our nanoparticle films can be changed by post-annealing treatment. Fig. 5 (a) shows the mobility change of five devices by post-annealing treatment. It is very clear that the electron mobility increased by post-annealing treatment in all the devices. Moreover, the mobility variation depending on the channel length was reduced remarkably by postannealing treatment. Before annealing, the mobility was largely varied from $2.5 \times 10^{-4}$ $\mathrm{cm}^{2} / \mathrm{Vs}$ to $1.1 \times 10^{-3} \mathrm{~cm}^{2} / \mathrm{Vs}$. After annealing treatment at $180^{\circ} \mathrm{C}$, the mobility values settled in the range of $0.023-0.027 \mathrm{~cm}^{2} / \mathrm{Vs}$ irrespective of the channel length. The hysteresis was also largely reduced by annealing as shown in Fig. 5 b. Although there is somewhat large variation, the threshold voltages $\left(\mathrm{V}_{\text {th }}\right)$ tend to decline and the on/off ratios $\left(\mathrm{I}_{\mathrm{on}} / \mathrm{I}_{\mathrm{off}}\right)$ clearly increase as the annealing temperature rises (S4).

Those changes with post-annealing treatment can be interpreted by the change of the film morphology. Fig. 6 shows film morphologies before and after annealing treatment observed by AFM. We observed low coverage regions intentionally in order to identify the contact layers with the gate dielectric which are considered the most important layers for the charge movement. For a non-treated film (before annealing, Fig. 6 a), we found that rod-shaped particles with diameters of smaller than $100 \mathrm{~nm}$ are 
assembled irregularly. This observation is in good agreement with the results of DLS and TEM measurements shown in Fig. 3. After annealed at $100^{\circ} \mathrm{C}$ (Fig. 6 b), the particle shapes were mainly changed together with slight growth of the particle size, hence the grain size of the film. As the annealing temperature rises to $140^{\circ} \mathrm{C}$ and $180^{\circ} \mathrm{C}$ the grain sizes of the film are also grown as shown in Fig. 6 (c) and (d). This grain growth is also confirmed by the measurement of XRD patterns (Fig. 7). The full width at half maximum (FWHM) of 001 peaks at near $3^{\circ}$ decreased largely by annealing treatment (Fig. 7 b) which means the size of crystalline domains increased. This increase of domain sizes means the reduction of grain boundaries which can lead to the performance enhancement - increased mobility and on/off ratio, decreased threshold voltage and hysteresis - effectively. The grain growth, however, is not as large as expected. In the case of vacuum deposited films, the grain size increased dramatically by post-annealing treatment up to a few micrometers which results in very high electron mobility of $2.1 \mathrm{~cm}^{2} / \mathrm{Vs}[32]$. In this case, the increased grain size is still an order of several hundred nanometers which results in somewhat low mobility of $0.027 \mathrm{~cm}^{2} / \mathrm{Vs}$. Therefore, it will be future works to increase the grain size dramatically by optimization various experimental conditions including the post treatment.

\section{Summary}

We demonstrated that our new approach to realize wet-processes of small molecular organic semiconductors, utilizing colloids prepared by laser ablation technique, is very successful. Highly stable PTCDI-C13 colloids of $0.1 \mathrm{wt} \%$ concentration were prepared successfully by laser ablation technique in acetonitrile 
whose mean particle size was smaller than $50 \mathrm{~nm}$. By simple drop-casting of prepared PTCDI-C13 colloids, typical n-type OTFTs with good saturation properties were fabricated successfully. The highest electron mobility was $1.1 \times 10^{-3} \mathrm{~cm}^{2} / \mathrm{Vs}$ before postannealing treatment and increased up to $0.027 \mathrm{~cm}^{2} / \mathrm{Vs}$ by post-annealing treatment. There is still large room for the enhancement of the device performance in this approach by optimizing various experimental conditions such as the particle size of colloids and also by adopting proper post-treatment leading to large increase of grain size. This new approach can be also widely applied to other small molecular organic semiconductors with high device performance and low solubility.

\section{Acknowledgement}

This work was supported by Program for Fostering Regional Innovation in Nagano, granted by the Ministry of Education, Culture, Sports, Science and Technology (MEXT), Japan. 
References

[1] C. Wöll (Ed.), Physical and Chemical Aspects of Organic Electronics: From Fundamentals to Functional Devices, WILEY-VCH, Weinheim, 2009.

[2] W. L. Leong, N. Mathews, B. Tan, S. Vaidyanathan, F. Dötz, S. Mhaisalkar, J. Mater. Chem. 21 (2011) 5203.

[3] G. Gelinck, P. Heremans, K. Nomoto, T. D. Anthopoulos, Adv. Mater. 22 (2010) 3778.

[4] M. E. Roberts, A. N. Sokolov, and Z. Bao, J. Mater. Chem. 19 (2009) 3351.

[5] A. R. Murphy, J. M. J. Fréchet, Chem, Rev. 107 (2007) 1066.

[6] M. Muccini, Nat. Mater. 5 (2006) 605.

[7] Q. Meng, H. Dong, W. Hu, D. Zhu, J. Mater. Chem. 21 (2011) 11708.

[8] D. Braga, G. Horowitz, Adv. Mater. 21 (2009) 1473.

[9] H. Klauk (Ed.), Organic Electronics: Materials, Manufacturing and Applications, WILEY-VCH, Weinheim, 2006.

[10] M. Berggren, D. Nilsson, N. D. Robinson, Nat. Mater. 6 (2007) 3.

[11] S. E. Shaheen, D. S. Ginley, G. E. Jabbour, MRS Bull. 30 (2005) 10.

[12] H. Sirringhaus, Adv. Mater. 17 (2005) 2411.

[13] S. R. Forrest, Nature 428 (2004) 911.

[14] F. Garnier, R. Hajlaoui, A. Yassar, P. Srivastava, Science 265 (1994) 1684.

[15] A. Facchetti, Chem. Mater. 23 (2011) 733.

[16] A. Afzali, C. D. Dimitrakopoulos, T. O. Graham, Adv. Mater. 15 (2003) 2066.

[17] T. -H. Chuang, H. -H. Hsieh, C. -K. Chen, C. -C. Wu, C. -C. Lin, P. -T. Chou, T. -H. Chao, T. J. Chow, Org. Lett. 10 (2008) 2869. 
[18] J. E. Anthony, Angew. Chem. Int. Ed. 47 (2008) 452.

[19] A. Luzio, C. Musumeci, C. R. Newman, A. Facchetti, T. J. Marks, B. Pignataro, Chem. Mater. 33 (2011) 1061.

[20] S. Allard, M. Forster, B. Souharce, H. Thiem, U. Scherf, Angew. Chem. Int. Ed. 47 (2008) 4070.

[21] P. Gao, D. Beckmann, H. N. Tsao, X. Feng, V. Enkelmann, M. Baumgarten, W. Pisula, K. Müllen, Adv. Mater. 21 (2009) 213.

[22] T. Izawa, E. Miyazaki, K. Takimiya, Chem. Mater. 21 (2009) 903.

[23] H. Yan, Y. Zheng, R. Blache, C. Newman, S. Lu, J. Woerle, A. Facchetti, Adv. Mater. 20 (2008) 3393.

[24] C. Piliego, D. Jarzab, G. Gigli, Z. Chen, A. Facchetti, M. A. Loi, Adv. Mater. 21 (2009) 1573.

[25] H. N. Tsao, D. Cho, J. W. Andreasen, A. Rouhanipour, D. W. Breiby, W. Pisula, K. Müllen, Adv. Mater. 21 (2009) 209.

[26] A. Salleo, Mater. Today 10 (2007) 38.

[27] B. S. Ong, Y. Wu, Y. Li, P. Liu, H. Pan, Chem. Eur. J 14 (2008) 4766

[28] Th. B. Singh, S. Erten, S. Günes, C. Zafer, G. Turkmen, B. Kuban, Y. Teoman, N. S. Sariciftci, S. Icli, Org. Electron. 7 (2006) 480

[29] B. A. Jones, A. Facchetti, M. R. Wasielewski, T. J. Marks, J. Am. Chem. Soc. 129 (2007) 15259

[30] R. P. Ortiz, H. Herrera, R. Blanco, H. Huang, A. Facchetti, T. J. Marks, Y. Zheng, J. L. Segura, J. Am. Chem. Soc. 132 (2010) 8440

[31] H. E. Katz, J. G. Laquindanum, A. J. Lovinger, Chem. Mater. 10 (1998) 633

[32] S. Tatemichi, M. Ichikawa, T. Koyama, Y. Taniguchi, Appl. Phys. Lett. 89 (2006) 
112108

[33] X. Zhan, A. Facchetti, S. Barlow, T. J. Marks, M. A. Ratner, M. R. Wasielewski, S. R. Marder, Adv. Mater. 23 (2011) 268

[34] Y. Wen, Y. Liu, C. Di, Y. Wang, X. Sun, Y. Guo, J. Zheng, W. Wu, W. Ye, G. Yu, Adv. Mater. 21 (2009) 1631

[35] D. J. Gundlach, K. P. Pernstich, G. Wilckens, M. Grüter, S. Haas, B. Batlogg, J. Appl. Phys. 98 (2005) 064502

[36] H. -G. Jeon, J. Hattori, S. Kato, N. Oguma, N. Hirata, Y. Taniguchi, M. Ichikawa, J. Appl. Phys. 108 (2010) 124512

[37] B. A. Jones, A. Facchetti, M. R. Wasielewski, T. J. Marks, Adv. Funct. Mater. 18 (2008) 1329

[38] Y. Tamaki, T. Asahi, H. Masuhara, Appl. Surf. Sci. 168 (2000) 85

[39] T. Sugiyama, T. Asahi, H. Takeuchi, H. Masuhara, Jpn. J. Appl. Phys. 45 (2006) 384

[40] S. Kita, S. Masuo, S. Machida, A. Itaya, Jpn. J. Appl. Phys. 45 (2006) 6501

[41] H. -G. Jeon, T. Sugiyama, H. Masuhara, T. Asahi, Jpn. J. Appl. Phys. 46 (2007) L733.

[42] H. -G. Jeon, S. Ryo, T. Sugiyama, I. Oh, H. Masuhara, T. Asahi, Chem. Lett. 36 (2007) 1160.

[43] H. -G. Jeon, T. Sugiyama, H. Masuhara, T. Asahi, J. Phys. Chem. C 111 (2007) 14658.

[44] R. J. Chesterfield, J. C. McKeen, C. R. Newman, P. C. Ewbank, D. A. da Silva Filho, J. -L. Brédas, L. L. Miller, K. R. Mann, C. D. Frisbie, J. Phys. Chem. B $108(2004) 19281$. 
[45] K. Balakrishnan, A. Datar, R. Oitker, H. Chen, J. Zuo, L. Zang, J. Am. Chem. Soc. $127(2005) 10496$.

[46] A. L. Briseno, S. C. B. Mannsfeld, C. Reese, J. M. Hancock, Y. Xiong, S. A. Jenekhe, Z. Bao, Y. Xia, Nano Lett. 7 (2007) 2847. 


\section{FIGURE CAPTIONS}

Fig. 1. (a) Molecular structure of PTCDI-C13, (b) $0.01 \mathrm{wt} \%$ PTCDI-C13/acetonitrile mixture before and after laser irradiation, and (c) the change of the absorption spectrum of a $0.01 \mathrm{wt} \%$ PTCDI-C13/acetonitrile colloid as a function of elapsed time until 10 days (afIrr_10d) after formation of the colloid (afIrr_soon).

Fig. 2. (a) Absorption spectra of $0.01,0.05$, and $0.1 \mathrm{wt} \%$ colloids measured in a quartz cell with $1 \mathrm{~mm}$ optical path length, and (b) the change of absorbance at the peak of 470 $\mathrm{nm}$ as a function of the concentration.

Fig. 3. (a) Size distribution of $0.01 \%$ PTCDI-C13 colloid in acetonitrile measured by DLS technique, and b) a TEM image of PTCDI-C13 colloidal particles.

Fig. 4. (a) A PTCDI-C13 nanoparticle film deposited by simple drop-casting of the prepared colloid, and representative (b) output characteristics and (c) transfer characteristics of its devices.

Fig. 5. (a) Mobility change of five devices by post-annealing, and b) representative transfer characteristics after post-annealing treatment at $180^{\circ} \mathrm{C}$.

Fig. 6. AFM images of (a) a non-treated film and annealed films at (b) $100^{\circ} \mathrm{C}$, (c) $140^{\circ} \mathrm{C}$, and (d) $180^{\circ} \mathrm{C}$. Scale bars indicate $500 \mathrm{~nm}$. 
Fig. 7. (a) XRD patterns of PTCDI-C13 nanoparticle films before and after postannealing treatment, and b) the change of FWHM of 001 peaks as a function of annealing temperature. 

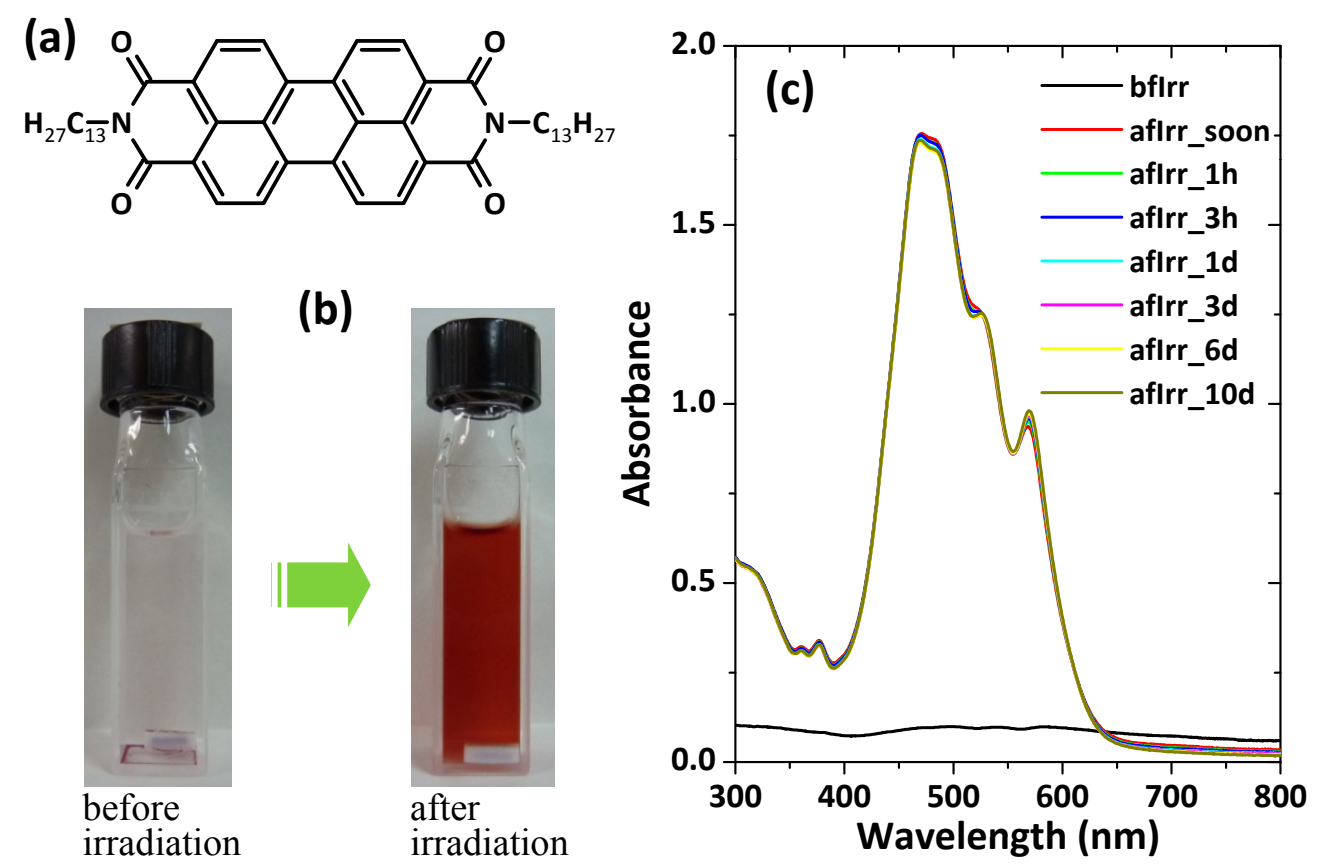

Figure 1. 

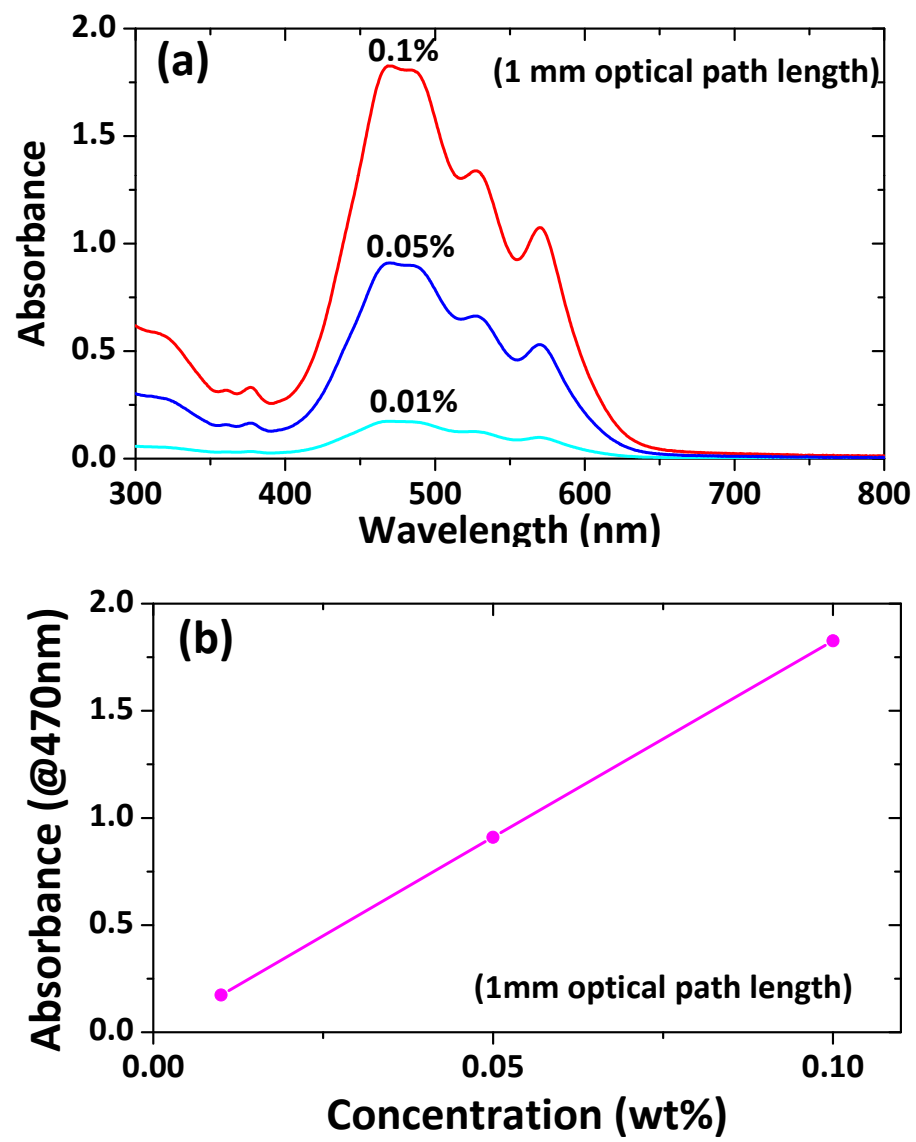

Figure 2. 


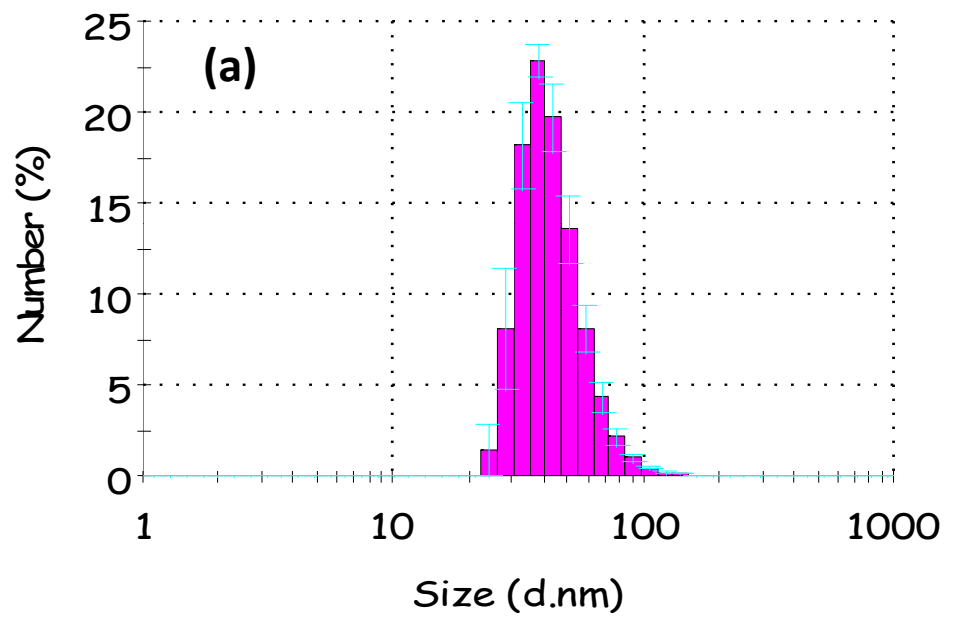

(b)

Figure 3. 

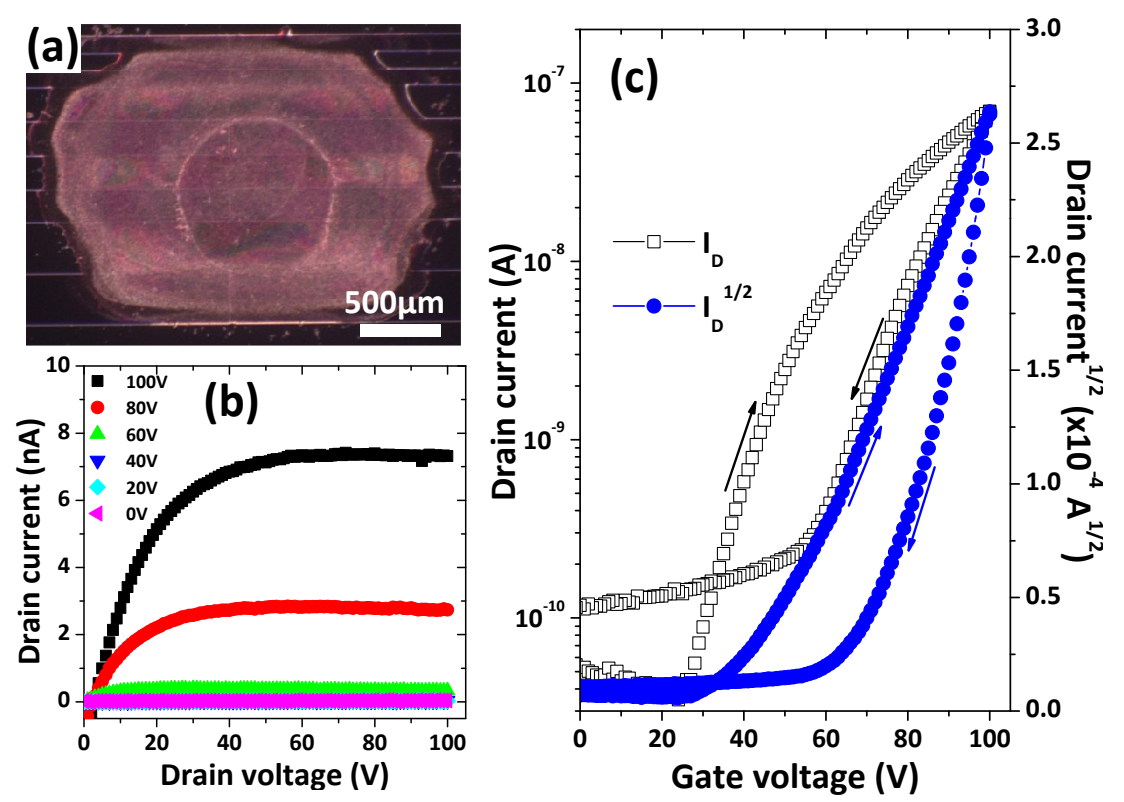

Figure 4. 

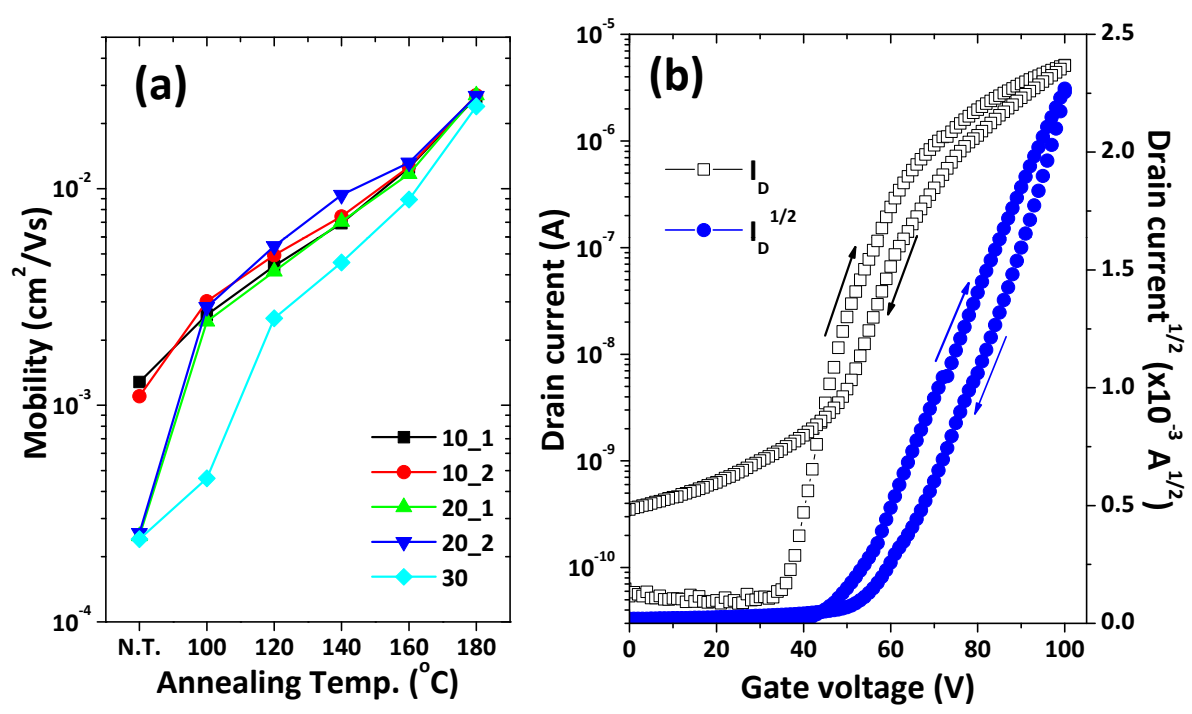

Figure 5. 


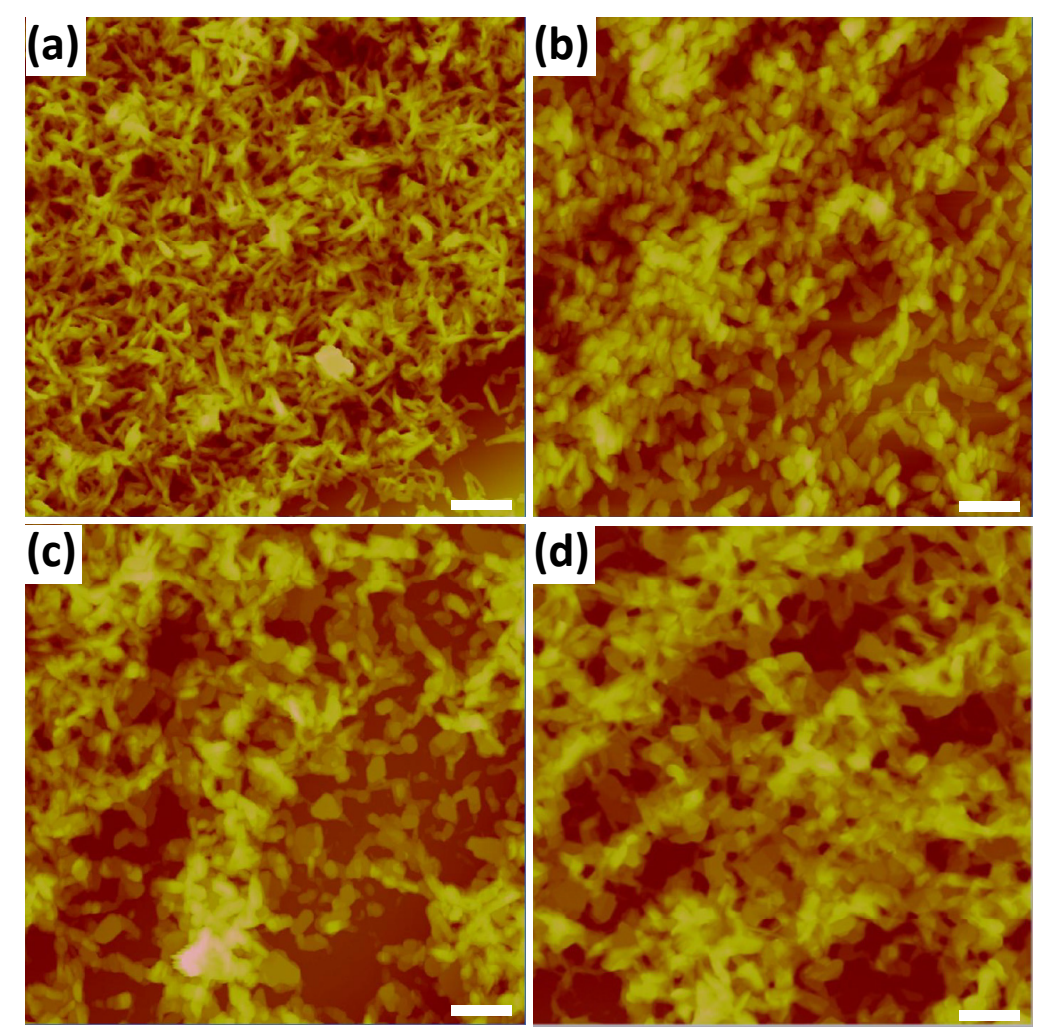

Figure 6. 

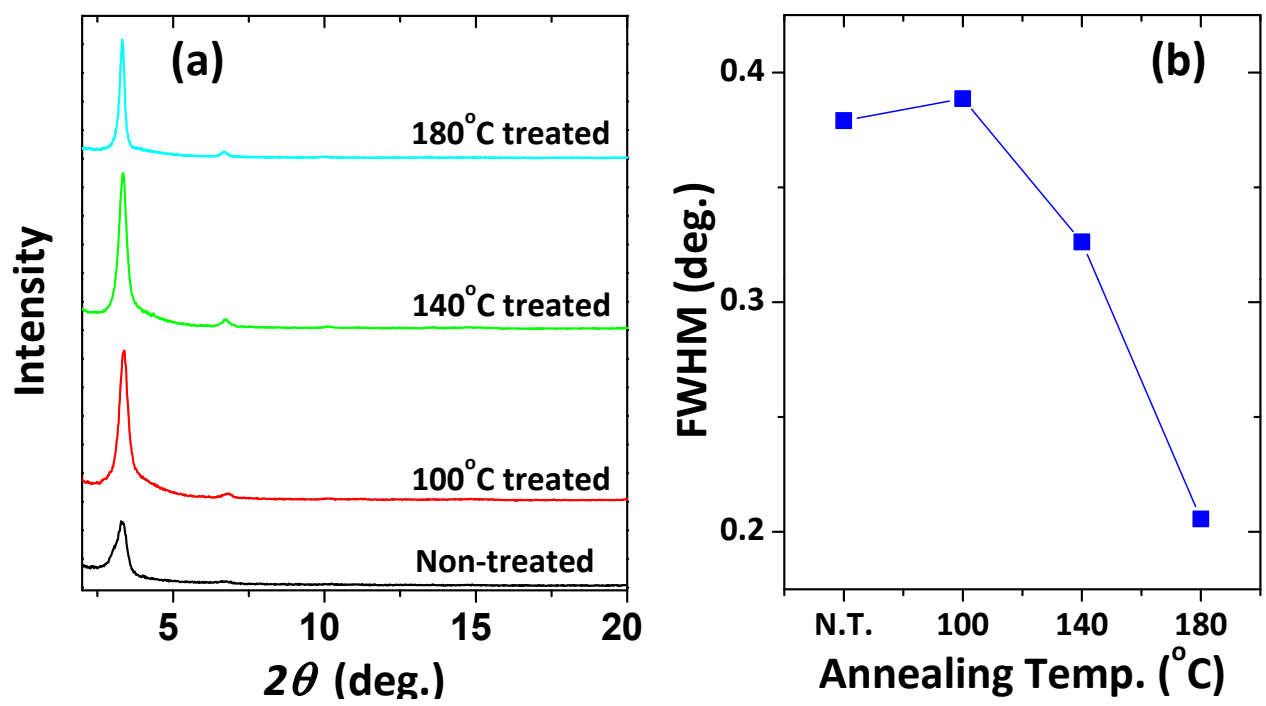

Figure 7. 


\section{Supporting Information}

S1. We have tried to make colloids in water, but we observed decomposition of PTCDI-C13 during the laser irradiation in water which is confirmed by FT-IR and UV-vis measurement (Fig. S1 a,b). We can see that FT-IR (in water in Fig. S1 a) and UV-vis spectra (in Water in Fig. S1 b) of PTCDI-C13/water mixture after laser irradiation are clearly different those of raw PTCDI-C13 (Raw in Fig. S1 a) and a vacuum deposited film (VacDepFilm in Fig. S1 b). The notable differences between two FT-IR spectra are highlighted with dotted colour-circles. Cast films prepared from that PTCDI-C13/water mixture did not show any transistor properties. We think that hydrolysis of PTCDI-C13 probably occurs during laser irradiation in water.

In contrast, for the case of acetonitrile colloid, the spectral shape (in Acetonitrile in Fig. $\mathrm{S} 1 \mathrm{~b}$ ) is rather closer to that of the vacuum deposited film which can be presumed as a benchmark spectrum of crystalline colloids of PTCDI-C13 except for interactions between the crystalline grains and grain orientations (if exist) in the films. Therefore, we currently think that there seems to be no harmful chemical decomposition in PTCDI-C13/acetonitrile mixture during laser irradiation.
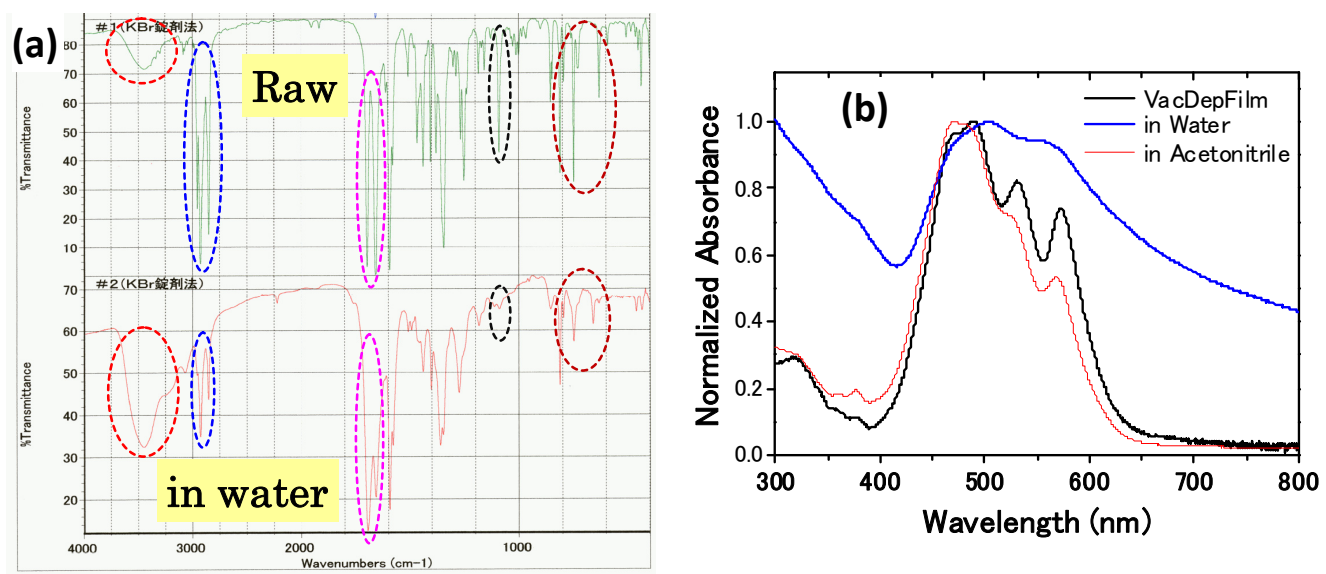

Figure S1. (a) FT-IR spectra of raw PTCDI-C13 (Raw) and laser irradiated PTCDI-C13 in water, and (b) UV-vis spectra of a vacuum deposited film (VacDepFilm), PTCDI-C13/water mixture (in Water), and PTCDI-C13/acetonitrile mixture (in Acetonitrile) after laser irradiation. 
S2. DLS measurement of colloids

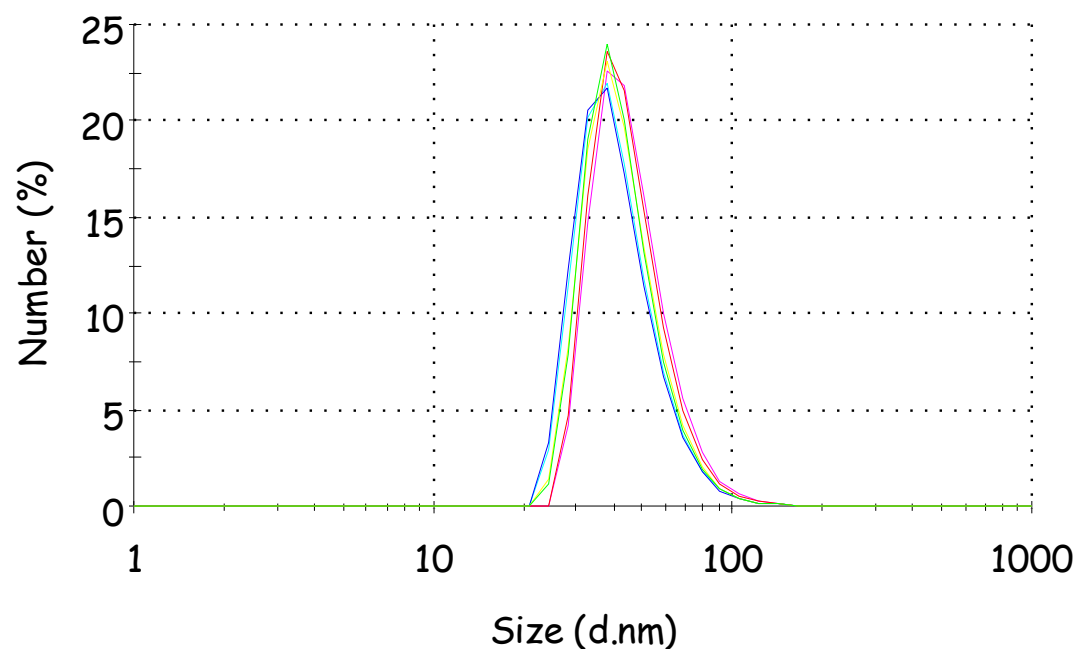

Figure S2. Size distribution of $0.01 \%$ PTCDI-C13 colloid in acetonitrile measured six times with separate batches. 
S3. Zeta potential measurement of colloids.

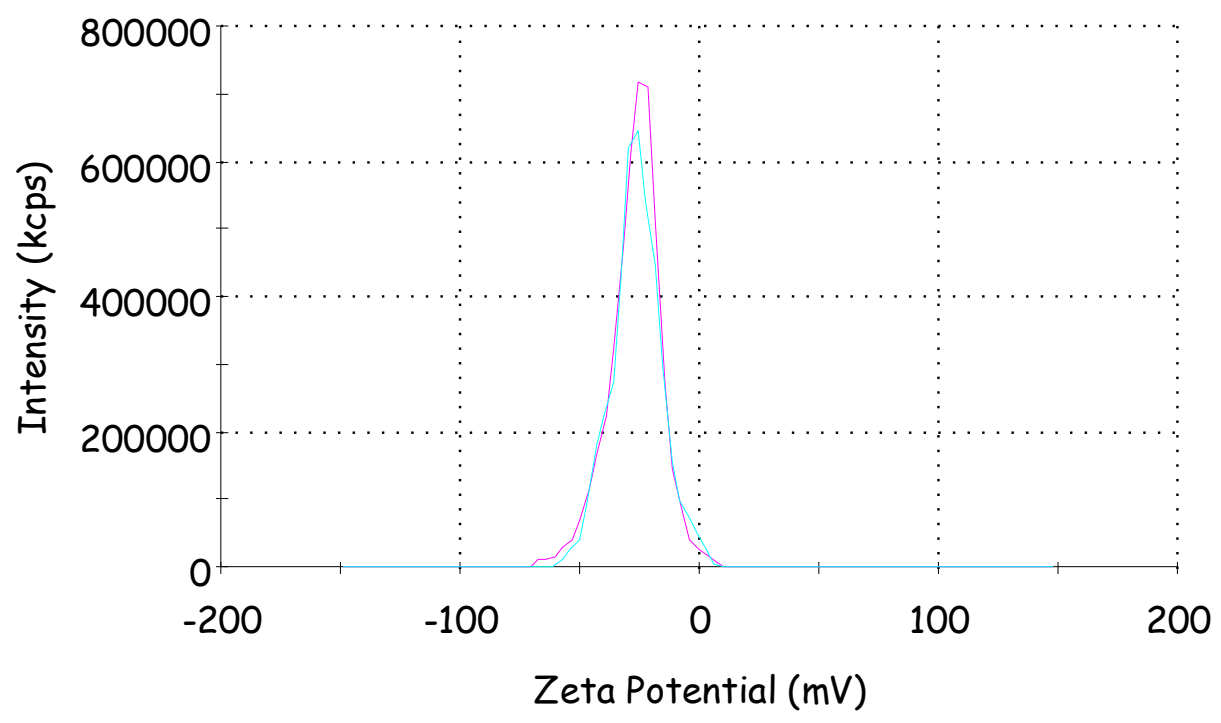

Figure S3. Zeta potential distribution of $0.01 \%$ PTCDI-C13 colloid in acetonitrile measured twice with separate batches. 
S4. Post-annealing effect
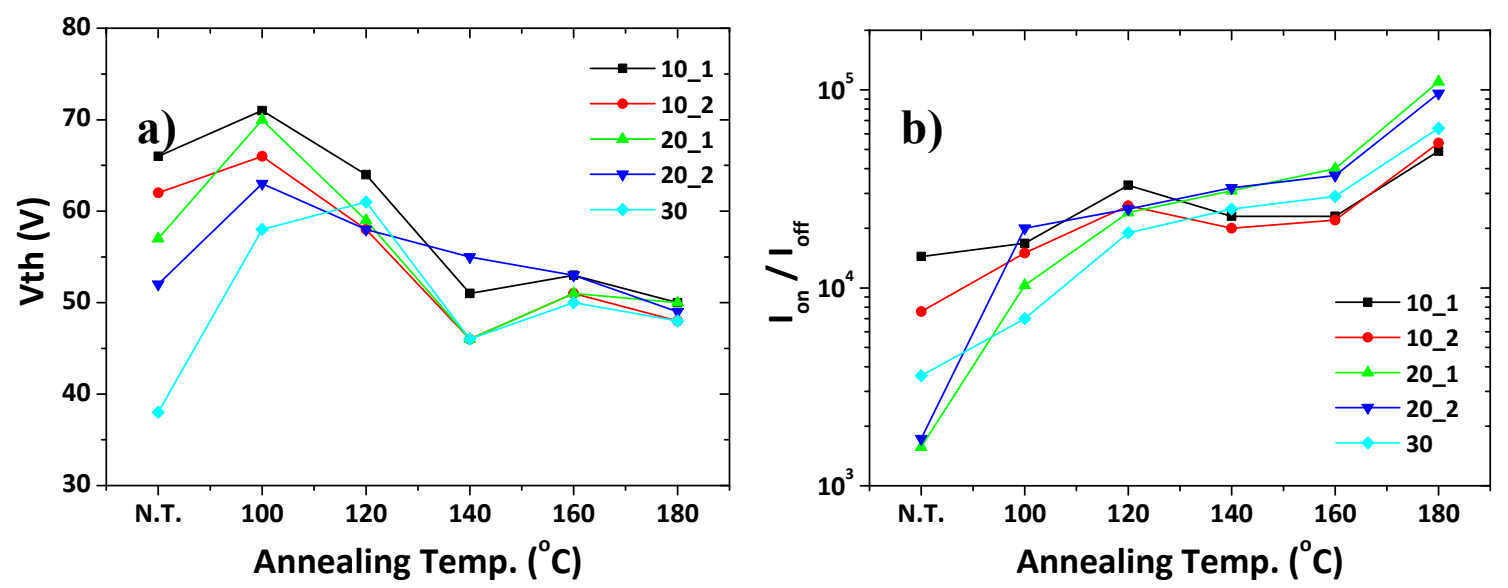

Figure S4. Changes of a) the threshold voltages and b) the on/off ratios as a function of annealing temperature. 